\title{
Längere Pause befreit nicht von Notdienstpflicht
}

_ Nach einem Urteil des Bundessozialgerichts (BSG) kann ein Arzt nicht von der Teilnahme am vertragsärztlichen Bereitschaftsdienst befreit oder ausgeschlossen werden, weil er über einen längeren Zeitraum nicht teilgenommen hat (Az.: B 6 KA 41/14 R).

Ein Facharzt für psychotherapeutische Medizin hatte von der KV eine Befreiung vom Notdienst erhalten, die jedoch 2007 nach mehreren Jahren wider- rufen wurde, da sich die Notfalldienstordnung geändert hatte. Das BSG bestätigte, dass Ärzte für psychotherapeutische Medizin dieselbe Verpflichtung zur Mitwirkung hätten wie alle anderen Vertragsärzte. Die Eignung könne der Kläger durch Fortbildung wiedergewinnen.

\section{MMW-KOMMENTAR}

Bisher war es in vielen KVen durchaus üblich, vorwiegend psychotherapeutisch tätig Ärzte, zu denen oft auch Hausärzte zählen, von der Teilnahme am Bereitschaftsdienst zu befreien. Dem zunehmenden Hausärztemangel dürfte es geschuldet sein, dass viele KVen nun doch auf alle personellen Ressourcen zurückgreifen und unter Hinweis auf die einschlägige Rechtsprechung auch hochspezialisierte Kollegen für den Dienst heranziehen. Diesem Umstand trägt dieses BSG-Urteil nunmehr bundeseinheitlich und grundsätzlich Rechnung.

\section{So rechnen Sie Notfallbehandlungen korrekt ab}

Im organisierten Not(fall)dienst kann der Erstkontakt in Abhängigkeit von Wochentag und Uhrzeit abgerechnet werden. Dafür stehen die Nrn. 01210 und 01212 EBM zur Verfügung (Tab. 1). Gibt es im gleichen Behandlungsfall einen weiteren Arzt-Patienten-Kontakt im Notfall oder im organisierten Notdienst, kommen die Nrn. 01214, 01216 oder 01218 zum Ansatz. Die Berechnung dieser fünf Nrn. erfordert grundsätzlich die Angabe der Uhrzeit. Neben den Notfallpauschalen ausgeschlossen sind lediglich Beratungs-, Gesprächs- und Erörterungsleistungen.

\section{MMW-KOMMENTAR}

Ist für die Notfallbehandlung ein Hausbesuch notwendig, kommt die Nr. 01418 zzgl. Kilometerpauschale zum Ansatz. Gerade bei solchen Notfalleinsätzen ist die Abrechnung der Nr. 01220 für künstliche Beatmung und/ oder extrathorakale Herzmassage denkbar. Die mit 105,49 Euro bewertete Leistung muss nicht zwangsläufig mit einer Intubation verbunden sein. Hierfür gibt es ausdrücklich den Zuschlag nach Nr. 01221. Muss der Arzt etwa

\section{Tab. 1 Häufige Leistungen im vertragsärztlichen Bereitschaftsdienst}

\begin{tabular}{|c|c|c|}
\hline EBM & Leistungsbeschreibung & Euro \\
\hline 01210 & Notfallpauschale bei Inanspruchnahme zwischen 7 und 19 Uhr & 13,05 \\
\hline 01212 & $\begin{array}{l}\text { Notfallpauschale zwischen } 19 \text { und } 7 \text { Uhr, samstags, sonntags, an Feiertagen, } \\
\text { am 24. oder 31.12. }\end{array}$ & 20,03 \\
\hline 01214 & Notfallkonsultationspauschale I zwischen 7 und 19 Uhr & 5,14 \\
\hline 01216 & $\begin{array}{l}\text { Notfallkonsultationspauschale II zwischen } 19 \text { und } 22 \text { Uhr bzw. } 7 \text { und } 19 \text { Uhr } \\
\text { samstags, sonntags, an Feiertagen, am 24. oder 31.12. }\end{array}$ & 14,38 \\
\hline 01218 & $\begin{array}{l}\text { Notfallkonsultationspauschale III zwischen } 22 \text { und } 7 \text { Uhr, bzw. } 19 \text { und } 7 \text { Uhr } \\
\text { samstags, sonntags, an Feiertagen, am 24. oder 31.12. }\end{array}$ & 17,46 \\
\hline 01418 & Besuch im organisierten Not(fall)dienst & 79,91 \\
\hline 01440 & $\begin{array}{l}\text { Verweilen außerhalb der Praxis ohne Erbringung weiterer Gebührenord- } \\
\text { nungspositionen, wegen der Erkrankung erforderlich, je } 30 \text { Minuten }\end{array}$ & 25,27 \\
\hline 02100 & Infusion, mindestens 10 Minuten & 5,85 \\
\hline 03330 & Spirografische Untersuchung & 6,16 \\
\hline 32025 & Glukosebestimmung & 1,60 \\
\hline
\end{tabular}

auf das Eintreffen des Krankentransportwagens warten, kann je vollendete 30 Minuten die Nr. 01440 berechnet werden. Wird dem Patienten eine Infusion verabreicht, die mindestens 10 Minuten läuft, kommt die Nr. 02100 zum Ansatz - dann allerdings ist die
Verweilgebühr nicht mehr möglich. Weitere denkbare Leistungen beim Vorhandensein der entsprechenden Utensilien wären die Nr. 03330 für eine spirografische Untersuchung sowie Ziffern des Akutlabors, etwa die Nr. 32025 für die Glukosebestimmung. 